\title{
Clinical Guideline Highlights for the Hospitalist: Evaluation and Management of Well-Appearing Febrile Infants 8 to 60 Days Old
}

\author{
Erin E Shaughnessy, MD, MSHCM ${ }^{1 *}$, Samir S Shah, MD, MSCE²
}

${ }^{1}$ University of Alabama-Birmingham, Birmingham, Alabama; ${ }^{2}$ Cincinnati Children's Hospital Medical Center and the University of Cincinnati College of Medicine, Cincinnati, Ohio.

GUIDELINE TITLE: Evaluation and Management of WellAppearing Febrile Infants 8 to 60 Days Old

RELEASE DATE: August 1, 2021

PRIOR VERSION(S): $n / a$

DEVELOPER: American Academy of Pediatrics
FUNDING SOURCE: American Academy of Pediatrics TARGET POPULATION: Well-appearing, otherwise healthy infants with fever, ages 8 to 60 days, excluding those with prematurity (<37 wk gestation), focal bacterial infections except acute otitis media, high suspicion for herpes simplex virus (vesicles), clinical bronchiolitis nvasive bacterial infections (IBI; ie, bacterial meningitis, bacteremia) are an uncommon but potentially devastating occurrence in young febrile infants. The challenge for clinicians is that physical examination cannot reliably exclude such infections. Thus, these infants have historically received comprehensive emergency department evaluation, including routine cerebrospinal fluid (CSF) assessment, and, often, required hospitalization for parenteral antibiotic administration while awaiting CSF culture results. The new American Academy of Pediatrics (AAP) guidelines were necessary given changing bacteriology, advances in diagnostic testing, greater insight into the differential risk of poor outcomes by site of infection, and better appreciation of the potential harms of unnecessary care and interventions. ${ }^{1}$ The 21 recommendations apply to well-appearing febrile infants 8 to 60 days of age, with recommendations stratified by age group, and exclude infants with certain conditions, including prematurity, focal bacterial infection, congenital or chromosomal abnormalities, and bronchiolitis. Four key recommendations are highlighted.

\section{KEY RECOMMENDATIONS FOR THE HOSPITALIST}

Recommendation 1: Diagnostic evaluation. For all age groups, blood culture and urinalysis (UA) are routinely recommended. For infants 8 to 21 days old, urine culture is routinely recommended. For older infants, urine culture is recommended if the UA is positive. All specimens for culture should be obtained via catheterization or suprapubic aspiration.

\section{Infants 8 to 21 days old}

- May assess inflammatory markers (grade B, weak).

- Should obtain CSF for analysis and culture (grade A, strong).

Infants 22 to 28 days old

- Should assess inflammatory markers (grade B, strong).

*Corresponding Author: Erin E Shaughnessy, MD, MSHCM;

Email: eshaughnessy@uabmc.edu; Twitter: @ErinShaughness4.

Received: July 12, 2021; Revised: July 30, 2021; Accepted: August 2, 2021

C 2021 Society of Hospital Medicine DOI 10.12788/jhm.3696
- May obtain CSF for analysis and culture even if no inflammatory marker obtained is abnormal (grade B, moderate).

- Should obtain CSF for analysis and culture if any inflammatory marker obtained is abnormal (procalcitonin $>0.5 \mathrm{ng} /$ $\mathrm{mL}$ [preferred]; C-reactive protein $>20 \mathrm{mg} / \mathrm{L}$; absolute neutrophil count $>4000-5200 / \mathrm{mm}^{3}$; or temperature $>38.5^{\circ} \mathrm{C}$ ) (grade B, moderate).

\section{Infants 29 to $\mathbf{6 0}$ days old}

- Should assess inflammatory markers (grade B, moderate).

- May obtain CSF for analysis and culture if any inflammatory marker is abnormal, (grade C, weak).

- Need not obtain CSF for analysis if all inflammatory markers obtained are normal (grade B, moderate).

\section{Recommendation 2: Initial disposition decision Infants $\mathbf{8}$ to 21 days old \\ - Admit (grade B, moderate).}

\section{Infants $\mathbf{2 2}$ to $\mathbf{2 8}$ days old}

- Admit if CSF analysis is abnormal, UA is positive (A, strong), or if CSF is not obtained or is uninterpretable (grade $B$, weak).

- May manage at home if UA is normal, inflammatory markers are normal, CSF is normal or enterovirus positive, family has received verbal and written home monitoring instructions for concerning signs that should prompt immediate return for care, follow-up plan for reevaluation in 24 hours is in place, and means of communication for change in clinical status has been established (grade B, moderate).

\section{Infants 29 to $\mathbf{6 0}$ days old}

- Admit if CSF analysis is abnormal (grade A strong).

- May hospitalize if any inflammatory marker obtained is abnormal (grade B, moderate).

- Should manage at home if all the following are present: CSF is normal, if obtained; UA is negative; all inflammatory markers obtained are normal; teaching is complete; follow-up plan for reevaluation in 24 hours is in place; and means of communication for change in clinical status has been established (grade B, moderate). 


\section{Recommendation 3: Empiric antimicrobial treatment Infants 8 to 21 days old}

- Should initiate parenteral antimicrobial therapy (grade A, strong).

- This recommendation is based on the high prevalence of $\mathrm{IBI}$ in this age category, and IBI may be present despite a negative UA and/or normal inflammatory markers.

\section{Infants $\mathbf{2 2}$ to $\mathbf{2 8}$ days old}

- Should initiate parenteral antimicrobial therapy if either CSF analysis suggests bacterial meningitis or UA is positive (grade A, strong).

- May administer parenteral antimicrobial therapy if any inflammatory marker is abnormal (grade $\mathrm{B}$, moderate).

- May administer parenteral antimicrobial therapy even if everything is reassuring (grade $B$, weak).

- Should administer parenteral antimicrobial therapy to infant who will be managed at home even if all evaluation is reassuring (grade $\mathrm{C}$, moderate).

\section{Infants 29 to 60 days old}

- Should start parenteral antimicrobials if CSF analysis suggests bacterial meningitis (grade A, strong).

- May use parenteral antimicrobials if any inflammatory marker is abnormal (grade B, moderate).

- Should initiate oral antimicrobial therapy if CSF is normal (if obtained), UA is positive, and no inflammatory markers obtained are abnormal (grade $B$, strong).

- Need not start antimicrobials if CSF is normal or enterovirus positive, UA is negative, and no inflammatory marker obtained is abnormal (grade B, moderate).

\section{Recommendation 4: Hospital discharge decision Infants 8 to 21 days AND Infants 22 to 28 days old}

- Discontinue antibiotics and discharge infant when culture results are negative for 24 to 36 hours (or positive only for contaminants), the infant is well or improving, and there are no other reasons for hospitalization (grade B, strong).

\section{Infants $\mathbf{2 9}$ to $\mathbf{6 0}$ days old}

- Although no specific parameters are given for infants without UTI, presumably the discharge criteria for younger infants would also apply for this group.

- For infants with UTI, discharge if blood and CSF cultures are negative, infant is well or improving, and no other reasons for hospitalization remain (grade $\mathrm{B}$, strong).

\section{CRITIQUE}

The guideline provides opportunities for safely doing less in a vulnerable population. For example, infants with UTIs may be managed differently (eg, often with oral antibiotics) from those with IBls, which represents an important change from conventional practice. ${ }^{2}$ Additional strengths are the incorporation of procalcitonin, which has emerged as the most accurate marker for risk stratification; ${ }^{3}$ and deemphasis of complete blood count results.

Multiple exclusions for relatively common scenarios represent missed opportunities for a more complete set of recommenda- tions for the febrile infant population. The decision to exclude infants in the first week of life is perplexing since infants 0 to 7 days old will receive CSF analysis, require admission, and generally be managed comparably to infants 8 to 21 days old. Infants with bronchiolitis are excluded; the absence of uniform guidance may perpetuate variability in management within and across institutions. Finally, exclusion of infants in whom perinatal or congenital herpes simplex virus is a consideration is not ideal. The requirement to consult separate guidance for herpes simplex virus evaluation fragments decision-making and may lead to inadvertent omissions of critical tests or treatment in at-risk infants.

\section{Methods in Preparing the Guideline}

The guideline working group included stakeholders from multiple specialties including general pediatrics, emergency medicine, hospital medicine, infectious diseases, and family medicine. In addition to published studies, the committee considered an Agency for Healthcare Research and Quality commissioned systematic review, as well as analyses of additional data solicited from previously published peer-reviewed studies. Once recommendations were formulated, additional input from physician focus groups and parents was solicited. Recommendations were rated based on strength of available evidence $(A, B, C, D, X)$ as well as assessment of the benefit/harm profile (strong, moderate, weak).

\section{Sources of Potential Conflicts of Interest or Bias}

The guideline writing group was predominantly male, though we note that the broader working group was diverse in gender and specialty. No significant conflicts of interest were noted.

\section{Generalizability}

The complexity of this guideline, including age stratification, multiple exclusions, and multistep processes could lead to challenges in implementation; a health information technology application (app) could substantially ease the difficulty of implementation at the point of care.

\section{AREAS IN NEED OF FUTURE STUDY}

Additional areas in need of guidance include neonates with bronchiolitis and fever and neonates with focal infection. For the former, there is an abundance of evidence; ${ }^{4}$ what is needed is consensus. For the latter, additional study is needed such as the role of inflammatory markers in stratifying infants with focal infection who need additional evaluation prior to treatment.

Disclosures: The authors have no conflicts to disclose.

References

1. Pantell RH, Roberts KB, Adams WG, et al; Subcommittee on Febrile Infants. Evaluation and management of well-appearing febrile infants 8-60 days old. Pediatrics. 2021; 148(2):e2021052228. https://doi.org/10.1542/peds.2021052228

2. Chang PW, Wang ME, Schroeder AR. Diagnosis and management of UTI in febrile infants age $0-2$ months: applicability of the AAP guideline. J Hosp Med. 2020;15(3): 176-180. https://doi.org/10.12788/jhm.3349

3. Wang ME, Srinivas N, McCulloh RJ. Clinical progress note: procalcitonin in the identification of invasive bacterial infections in febrile young infants. J Hosp Med. 2021; 16(3): 165-167. https://doi.org/10.12788/jhm.3451

4. Ralston $S$, Hill V, Waters A. Occult serious bacterial infection in infants younger than 60 to 90 days with bronchiolitis: a systematic review. Arch Pediatr Adolesc Med. 2011;165(10):951-956. https://doi.org/1 0.1001/archpediatrics.2011.155 